\title{
Linearity of the core correspondence
}

\author{
Dénes Pálvölgyi ${ }^{1}$ - Hans Peters ${ }^{2}$. \\ Dries Vermeulen ${ }^{2}$
}

\begin{abstract}
Bloch and de Clippel (J Econ Theory 145:2424-2434, 2010) characterized sets of balanced TU-games on which the core correspondence is linear by means of an equivalence relation. We characterize maximal regions on which the core correspondence is linear in four different ways. First, by finitely many linear equalities and inequalities; thus, the core is piecewise linear. Second, maximal linear regions coincide with closures of equivalence classes (in the sense of Bloch and de Clippel) that are maximal w.r.t. set inclusion. Third, maximal linear regions coincide with closures of equivalence classes of full dimension. Fourth, for every extreme point of the core of a game in the interior of a maximal linear region, the collection of tight core inequalities constitutes a basis.
\end{abstract}

Keywords Transferable utility games · Core correspondence · Piecewise linearity

\section{Introduction}

Since the graph of the core correspondence is defined by finitely many linear inequalities, this graph is a polyhedral set. It is therefore to be expected that the core correspondence is piecewise linear. Bloch and de Clippel (2010) introduced an equivalence relation based on the extreme points of the core to identify the classes of balanced games on which the core is additive. In this paper, using a result of Kuipers et al.

$\bowtie$ Dries Vermeulen

d.vermeulen@maastrichtuniversity.nl

1 Department of Mathematical Economics and Economic Analysis, Corvinus University of Budapest, MTA-BCE “Lendület” Strategic Interactions Research Group, Budapest, Hungary

2 Department of Quantitative Economics, Maastricht University, P.O. Box 616, 6200 MD Maastricht, The Netherlands 
(2010), we characterize those equivalence classes by finitely many linear inequalities. It follows that the core correspondence is indeed piecewise linear.

Additionally, we provide three alternative characterizations of the maximal regions on which the core correspondence is linear. First, such regions coincide with closures of equivalence classes of full dimension. Second, such regions coincide with closures of equivalence classes that are maximal w.r.t. set inclusion. Third, for every extreme point of the core of a game in the interior of a maximal region, the collection of tight core inequalities constitutes a basis.

\section{Preliminaries}

For subsets $X$ and $Y$ of $\mathbb{R}^{n}$ and $\alpha, \beta \in \mathbb{R}, \alpha X+\beta Y=\{\alpha x+\beta y \mid x \in X, y \in Y\}$. We write $N=\{1, \ldots, n\}$. For a convex set $X \subseteq \mathbb{R}^{k}$, let $H$ be the smallest affine subspace that contains $X$. The relative interior relint $(X)$ of $X$ is the collection of points $x \in X$ for which there is an open set $U \ni x$ in $\mathbb{R}^{k}$ such that $U \cap H$ is a subset of $X$. The set of extreme points of $X$ is denoted by ext $(X)$.

A transferable utility game, or game, is a pair $(N, v)$ where $N=\{1, \ldots, n\}$ is the set of players and $v$ is a function that assigns to each nonempty $S \subseteq N$ its worth $v(S) \in \mathbb{R}$. Such a nonempty set $S$ in $N$ is called a coalition. ${ }^{1}$ Throughout this paper we keep the player set $N$ fixed. To simplify notation we write $v$ instead of $(N, v)$ to denote a game.

A vector $x \in \mathbb{R}^{n}$ is an allocation. The $i$ th coordinate $x_{i}$ of the allocation $x$ represents the payoff to player $i \in N$. For coalition $S \subseteq N$, we write $x(S)=\sum_{i \in S} x_{i}$. An allocation $x$ is efficient for $v$ if it distributes the worth of the grand coalition among the players of the game $v$, i.e., if $x(N)=v(N)$. An efficient allocation $x$ is a core allocation for $v$ if

$$
x(S) \geq v(S) \text { for all } S \subseteq N .
$$

The set of core allocations, denoted by $C(v)$, is called the core of $v$. Bondareva (1962) and Shapley (1967) have shown that the core of a game is not empty precisely when the game is balanced. Therefore we do not define balancedness, but act as if it is identical to non-emptiness of the core.

It is well-known and easy to prove that for any two balanced games $v$ and $w$ and $0 \leq \alpha \leq 1$ we have $\alpha C(v)+(1-\alpha) C(w) \subseteq C(\alpha v+(1-\alpha) w)$. Here, $\alpha v+(1-\alpha) w$ is the game defined by $(\alpha v+(1-\alpha) w)(S)=\alpha v(S)+(1-\alpha) w(S)$ for every coalition $S$.

\section{Limit games for balanced games}

For an allocation $x \in \mathbb{R}^{n}$, a coalition $S$ with $x(S)=v(S)$ is called tight at $x$ in $v$. The collection of coalitions that are tight at $x$ in $v$ is denoted by $T(v, x)$. We say that

\footnotetext{
${ }^{1}$ Thus, the empty set is not a coalition. Although this is not entirely standard, it is convenient in the context of this paper.
} 
$w$ is a limit game for $v$ if for every extreme point $x \in C(v)$ there is an extreme point $y \in C(w)$ with $T(v, x) \subseteq T(w, y)$. The collection of limit games for $v$ is denoted by $L(v)$. For later reference we note that if $w \in L(v)$ and $w^{\prime} \in L(w)$, then $w^{\prime} \in L(v)$.

For a coalition $S \subseteq N, e^{S} \in \mathbb{R}^{N}$ is the vector with $e_{i}^{S}=1$ if $i \in S$ and $e_{i}^{S}=0$ otherwise. A set of coalitions $B$ is a basis if $\left\{e^{S} \mid S \in B\right\}$ is a basis for $\mathbb{R}^{n}$.

Let $v$ be a balanced game, let the set $B$ of coalitions be a basis, and let $T$ be a coalition with $T \notin B$. Note that, for every $x \in \operatorname{ext}(C(v))$, the set $T(v, x)$ contains a basis. We say that $B$ is feasible for $v$ if there is an extreme point $x \in C(v)$ such that $B \subseteq T(v, x)$. We say that $T$ is a neighbor of $B$ if there exists a basis $C$ that is feasible for $v$ such that $T \in C$ and $|B \cap C|=n-1$. We say that $T$ is spanned by $B$ if the unique numbers $\lambda(S)$ with $e^{T}=\sum_{S \in B} \lambda(S) e^{S}$ are nonnegative whenever $S \neq N .^{2}$

A feasible pair for $v$ is a pair $(B, T)$ where $B$ is a basis that is feasible for $v$, and coalition $T \notin B$ is a neighbor of $B$ or spanned by $B$.

For a balanced game $v$, we construct a system of linear inequalities of which the solution set will equal the polyhedral cone $L(v)$. Let $(B, T)$ be a feasible pair for $v$. Let $\lambda(S)$ for $S \in B$ be the unique real numbers such that

$$
e^{T}=\sum_{S \in B} \lambda(S) e^{S}
$$

The linear inequality generated by the pair $(B, T)$ is

$$
w(T) \leq \sum_{S \in B} \lambda(S) w(S)
$$

We denote the set of all inequalities we can generate by choosing different feasible pairs $(B, T)$ by $I(v)$. The following result is proved in Kuipers et al. (2010).

Theorem 3.1 Let $v$ be a balanced game. Then a game $w$ satisfies the inequalities in $I(v)$ if and only if $w \in L(v)$.

This result implies that $L(v)$ is a polyhedral cone in the space of all games.

\section{Linearity of the core correspondence}

Let $\mathcal{B}$ be the collection of balanced games. The core correspondence $C: \mathcal{B} \rightarrow \mathbb{R}^{N}$ is the correspondence that assigns to each game $v \in \mathcal{B}$ the set $C(v)$.

Let $L$ be a convex subset of $\mathcal{B}$. The core correspondence $C$ is linear on $L$ when for all $v, w \in L$ and every $0<\alpha<1$ we have

$$
C(\alpha v+(1-\alpha) w)=\alpha C(v)+(1-\alpha) C(w) .
$$

\footnotetext{
${ }^{2}$ Since the notion of spanning already has a meaning in the context of linear algebra, perhaps a better name would be "non-negatively spanned". However, we decided to stay with the terminology used in Kuipers et al. (2010).
} 
We identify the sets of games on which the core correspondence is linear. Our first result, Theorem 4.2, gives a sufficient condition for (1) to hold. This result is in fact equivalent to the Proposition in Bloch and de Clippel (2010). In the proof of Theorem 4.2 we use the following lemma. The lemma shows that in a limit game for a balanced game, no additional extreme core points can emerge.

Lemma 4.1 Let $v, w \in \mathcal{B}$ and $w \in L(v)$. Then, for every $y \in \operatorname{ext}(C(w))$ there is an $x \in \operatorname{ext}(C(v))$ such that $T(v, x) \subseteq T(w, y)$.

Proof For $v \in \mathcal{B}$ and $x \in \operatorname{ext}(C(v))$, let $R(v, x)$ be the points $z \in \mathbb{R}^{n}$ for which there is $\lambda(N) \in \mathbb{R}$ and for every $S \in T(v, x)$ there is a non-negative number $\lambda(S)$ such that

$$
z=\lambda(N) \cdot e^{N}+\sum_{S \in T(v, x)} \lambda(S) \cdot e^{S} .
$$

Since the sets $R(v, x)$ are the dual cones, they are of dimension $n$, intersections of sets $R(v, x)$ have empty interior, and the union of the sets $R(v, x)$ over all $x \in \operatorname{ext}(C(v))$ equals $\mathbb{R}^{n}$.

Now notice that $T(v, x) \subseteq T(w, y)$ implies that $R(v, x) \subseteq R(w, y)$. Thus, since $w \in L(v)$, the union of all sets $R(w, y)$ over those $y \in \operatorname{ext}(C(w))$ for which there is an $x \in \operatorname{ext}(C(v))$ with $T(v, x) \subseteq T(w, y)$ already equals $\mathbb{R}^{n}$. Hence, for every $y \in \operatorname{ext}(C(w))$ there is an $x \in \operatorname{ext}(C(v))$ such that $T(v, x) \subseteq T(w, y)$.

The following proposition says that for each balanced game the core correspondence is linear on the set of its limit games.

Theorem 4.2 Let $v \in \mathcal{B}$. Then $C(\cdot)$ is linear on $L(v)$.

Proof Let $w, w^{\prime} \in L(v)$ and $0<\alpha<1$. Let $\tilde{w}=\alpha w+(1-\alpha) w^{\prime}$ and let $z \in \operatorname{ext}(C(\tilde{w}))$. By Theorem 3.1 we know that $\tilde{w} \in L(v)$. By Lemma 4.1 there is an $x \in \operatorname{ext}(C(v))$ such that $T(v, x) \subseteq T(\tilde{w}, z)$. By definition of $L(v)$ there are $y \in \operatorname{ext}(C(w))$ and $y^{\prime} \in \operatorname{ext}\left(C\left(w^{\prime}\right)\right)$ such that $T(v, x) \subseteq T(w, y)$ and $T(v, x) \subseteq T\left(w^{\prime}, y^{\prime}\right)$. We claim that $z=\alpha y+(1-\alpha) y^{\prime}$, which completes the proof of the proposition. To show this claim, note that for all $S \in T(v, x)$ we have

$$
z(S)=\tilde{w}(S)=\alpha w(S)+(1-\alpha) w^{\prime}(S)=\alpha y(S)+(1-\alpha) y^{\prime}(S) .
$$

Since $T(v, x)$ contains a basis, this implies $z=\alpha y+(1-\alpha) y^{\prime}$.

\section{Maximal linear regions}

Call a convex subset $L$ of $\mathcal{B}$ a maximal linear region if the core correspondence is linear on $L$ but not linear on any convex subset $L^{\prime}$ of $\mathcal{B}$ with $L \subseteq L^{\prime}$ and $L \neq L^{\prime}$. In the remainder of this paper we consider the question which convex sets $L \subseteq \mathcal{B}$ are maximal linear regions.

Lemma 5.1 Let $L$ be a convex subset of $\mathcal{B}$ and let the core correspondence be linear on $L$. Then $L \subseteq L(v)$ for every $v \in \operatorname{relint}(L)$. 
Proof Take a game $v$ in the relative interior of $L$. Clearly, $v \in L(v)$. Take any $w \in L \backslash\{v\}$. We show that $w \in L(v)$. Take any $x \in \operatorname{ext}(C(v))$. We show that there exists a $y \in \operatorname{ext}(C(w))$ with $T(v, x) \subseteq T(w, y)$.

Since $v$ is an element of the relative interior of $L$, there is a $w^{\prime} \in L \backslash\{v\}$ and a $0<\alpha<1$ such that $v=\alpha w+(1-\alpha) w^{\prime}$. We first show that there are $y \in \operatorname{ext}(C(w))$ and $z \in \operatorname{ext}\left(C\left(w^{\prime}\right)\right)$ such that $x=\alpha y+(1-\alpha) z$.

By (1) there are $y \in C(w)$ and $z \in C\left(w^{\prime}\right)$ such that $x=\alpha y+(1-\alpha) z$. Suppose that $y \notin \operatorname{ext}(C(w))$. Take $p, q \in C(w)$ with $p \neq q$, and $\lambda \in(0,1)$ with $\lambda p+(1-\lambda) q=y$. Then $s=\alpha p+(1-\alpha) z \in C(v), t=\alpha q+(1-\alpha) z \in C(v)$, and $x=\lambda s+(1-\lambda) t$. Since $p \neq q$, we have $s \neq t$, which contradicts the assumption that $x \in \operatorname{ext}(C(v))$.

So, indeed $y \in \operatorname{ext}(C(w))$, and similarly $z \in \operatorname{ext}\left(C\left(w^{\prime}\right)\right)$. Let $S \in T(v, x)$. Then

$$
v(S)=x(S)=\alpha y(S)+(1-\alpha) z(S) \geq \alpha w(S)+(1-\alpha) w^{\prime}(S)=v(S) .
$$

Hence, $y(S)=w(S)$ and $z(S)=w^{\prime}(S)$, so that in particular $S \in T(w, y)$. It follows that $T(v, x) \subseteq T(w, y)$.

The following corollary is an immediate consequence.

Corollary 5.2 Let $L \subseteq \mathcal{B}$ be a maximal linear region. Then $L=L(v)$ for every $v \in \operatorname{relint}(L)$.

Proof By Lemma 5.1, $L \subseteq L(v)$ for any $v \in$ relint $(L)$. Since $C$ is linear on $L(v)$ by Proposition 4.2 and $L$ is a maximal region, it follows that $L=L(v)$.

Thus, for any maximal linear region $L$ there is a $v \in \mathcal{B}$ with $L=L(v)$. In other words, maximal linear regions are of the form $L(v)$. In the remainder of this paper we characterize the class of balanced games $v$ for which $L(v)$ is a maximal linear region. We first prove the following.

Lemma 5.3 Let $v, w \in \mathcal{B}$. Equivalent are

[1] $L(w) \subseteq L(v)$.

[2] $I(v) \subseteq I(w)$.

Proof A. We show [1] $\Longrightarrow$ [2]. Suppose that $L(w) \subseteq L(v)$. Take a feasible pair $(B, T)$ for $v$. We show that $(B, T)$ is also feasible for $w$, so that $I(v) \subseteq I(w)$. Since $(B, T)$ is feasible for $v$, there is an $x \in \operatorname{ext}(C(v))$ with $B \subseteq T(v, x)$, and $T \notin B$ is a neighbor of $B$ or spanned by $B$.

Since $w \in L(v)$, by definition there is a $y \in \operatorname{ext}(C(w))$ with $T(v, x) \subseteq T(w, y)$. Then also $B \subseteq T(w, y)$, and $T \notin B$ is a neighbor of $B$ or spanned by $B$. Hence, $(B, T)$ is feasible for $w$. It follows that $I(v) \subseteq I(w)$.

B. We show [2] $\Longrightarrow$ [1]. Suppose that $I(v) \subseteq I(w)$. Take $w^{\prime} \in L(w)$. Then, by Theorem 3.1, $w^{\prime}$ satisfies the inequalities in $I(w)$. Thus, since $I(v) \subseteq I(w), w^{\prime}$ also satisfies the inequalities in $I(v)$. Then, by Theorem 3.1, $w^{\prime} \in L(v)$. It follows that $L(w) \subseteq L(v)$.

We say that $L(v)$ is maximal (w.r.t. set inclusion) if there is no game $w \in \mathcal{B}$ with $L(v) \subseteq L(w)$ and $L(v) \neq L(w)$. We say that $I(v)$ is minimal (w.r.t. set inclusion) if there is no game $w \in \mathcal{B}$ with $I(w) \subseteq I(v)$ and $I(w) \neq I(v)$. 


\section{Theorem 5.4 Let $v \in \mathcal{B}$. Equivalent are}

[1] $L(v)$ is a maximal linear region.

[2] $L(v)$ is maximal.

[3] $I(v)$ is minimal.

[4] $L(v)$ is of full dimension $2^{n}-1$.

[5] for every extreme point of $C(v)$, the set $T(v, x)$ is a basis.

Proof A. We show [2] $\Longrightarrow[1]$. Suppose that $L(v)$ is not a maximal linear region. Then, due to Lemma 5.1, there is a $w \in \mathcal{B}$ with $L(v) \subseteq L(w)$ and $L(v) \neq L(w)$. Hence, $L(v)$ is not maximal.

B. We show [1] $\Longrightarrow[2]$. Suppose that $L(v)$ is not maximal. Take a game $w \in \mathcal{B}$ with $L(v) \subseteq L(w)$ and $L(v) \neq L(w)$. By Theorem 4.2, $L(v)$ is not a maximal linear region.

C. The equivalence of [2] and [3] follows directly from Lemma 5.3.

D. We show [4] $\Longrightarrow[2]$. Suppose that $L(v)$ is not maximal. Take $w \in \mathcal{B}$ with $L(v) \subseteq L(w)$ and $L(v) \neq L(w)$. By Theorem 3.1 we know that $L(v)$ is a polyhedral subset of the polyhedral set $L(w)$. Further, by Lemma 5.1, $L(v)$ has an empty intersection with the relative interior of $L(w)$. Hence, $L(v)$ does not have full dimension.

E. We show [2] $\Longrightarrow[4]$. Suppose that $L(v)$ is not of full dimension. Take a sequence $\left(v^{k}\right)_{k=1}^{\infty}$ outside $L(v)$ in $\mathcal{B}$ that converges to $v$. Since

$$
\{L(w) \mid w \in \mathcal{B}\}
$$

is finite, we may assume without loss of generality that there is a set $L$ with $L=L\left(v^{k}\right)$ for all $k$. Then, since $\left(v^{k}\right)_{k=1}^{\infty}$ converges to $v$, it follows from Theorem 3.1 that $v \in L$, and $L(v) \subseteq L$. However, $v^{k} \notin L(v)$, so $L(v) \neq L$. Hence, $L(v)$ is not maximal.

F. We show [5] $\Longrightarrow$ [2]. Suppose that for every extreme point of $C(v)$, the set $T(v, x)$ is a basis. Let $w \in \mathcal{B}$ be such that $L(v) \subseteq L(w)$. We show that $L(v)=L(w)$. Take an extreme point $x$ of $C(v)$. By Lemma 4.1 there is an extreme point $p(x)=y$ of $C(w)$ with $T(w, y) \subseteq T(v, x)$. Since $T(v, x)$ is a basis, and $T(w, y)$ must contain a basis, it is clear that $T(v, x)=T(w, y)$. This defines an injection $p$ of $\operatorname{ext}(C(v))$ into $\operatorname{ext}(C(w))$ that preserves tight inequalities. Moreover, since $v$ is a limit game of $w$, for every extreme point $y \in C(w)$ there is an extreme point $x \in C(v)$ with $T(w, y) \subseteq T(v, x)$. So, $p$ is one-to-one and surjective. Thus,

$$
\{T(v, x) \mid x \in \operatorname{ext}(C(v))\}=\{T(w, y) \mid y \in \operatorname{ext}(C(w))\}
$$

It follows that $I(v)=I(w)$, and hence also $L(v)=L(w)$.

G. We show [2] $\Longrightarrow[5]$. Suppose that there is an extreme point $x$ of $C(v)$ for which the set $T(v, x)$ is not a basis. We show that $L(v)$ is not maximal. Let $B$ be a basis contained in $T(v, x)$, and let $T$ be an element of $T(v, x)$ outside $B$. For $\varepsilon>0$, 
define the game $v(\varepsilon)$ by

$$
v(\varepsilon)(S)= \begin{cases}v(T)-\varepsilon & \text { if } S=T \\ v(S) & \text { otherwise. }\end{cases}
$$

Clearly $v(\varepsilon) \in \mathcal{B}$. Further, for sufficiently small $\varepsilon, v$ is a limit game of $v(\varepsilon)$. So, $L(v) \subseteq L(v(\varepsilon))$. However, we know that $x \in \operatorname{ext}(C(v(\varepsilon)))$, that $T(v(\varepsilon), x) \subseteq T(v, x)$, and that $T(v(\varepsilon), x) \neq T(v, x)$. It follows that $v(\varepsilon)$ is not a limit game of $v$. So, $L(v) \neq L(v(\varepsilon))$, and $L(v)$ is not maximal.

\section{Discussion}

In this section, we discuss a few immediate consequences of our results, and a few examples to illustrate those results. Theorem 7 and Corollary 11 in Kuipers et al. (2010) show that for a strictly convex game $v^{*}$, the set $I\left(v^{*}\right)$ is minimal. Thus, by Theorem 5.4, the class $\mathcal{C}=L\left(v^{*}\right)$ of all convex games is a maximal linear region.

Is any set $L(v)$ a maximal linear region? The answer to this question is negative. For instance, let $\mathcal{A}$ be the set of all additive games. Then it is easy to see that $\mathcal{A}=L(a)$ for any $a \in \mathcal{A}$. Take any $v \in \mathcal{B} \backslash \mathcal{A}$. Then the core correspondence is linear on the convex hull of $\mathcal{A} \cup\{v\}$, so that $\mathcal{A}$ is not a maximal linear region. A more sophisticated example is presented below.

Example A non-linear game $v$ whose set $L(v)$ is not a maximal linear region. Consider the three-person game given by

\begin{tabular}{r|ccccccc}
$S$ & $\{1\}$ & $\{2\}$ & $\{3\}$ & $\{1,2\}$ & $\{1,3\}$ & $\{2,3\}$ & $\{1,2,3\}$ \\
\hline$v(S)$ & 0 & 0 & 0 & 5 & 5 & 5 & 10
\end{tabular}

Note that the game $v$ is not additive. The game $v$ is convex, so that $L(v) \subseteq \mathcal{C}$. We already observed that $\mathcal{C}$ is a maximal linear region. We argue that $L(v) \neq \mathcal{C}$, so that $L(v)$ is not a maximal linear region.

The extreme elements of $C(v)$ are $a=(0,5,5), b=(5,0,5)$, and $c=(5,5,0)$. We can compute that

$$
\begin{aligned}
& T(v, a)=\{(1),(12),(13),(123)\} \\
& T(v, b)=\{(2),(12),(23),(123)\} \\
& T(v, c)=\{(3),(13),(23),(123)\}
\end{aligned}
$$

Now notice that $B=\{(12),(13),(123)\}$ is a feasible basis for $v$. Further, due to the vector equality

$$
e^{(1)}=e^{(12)}+e^{(13)}-e^{(123)}
$$

it follows that $T=(1)$ is spanned by $B$. So, $I(v)$ contains the inequality

$$
v(1) \leq v(12)+v(13)-v(123) .
$$


Since there are convex games $w$ for which $w(1)+w(123)>w(12)+w(13)$, it follows that $L(v) \neq \mathcal{C}$.

To take the analysis of this example one step further, notice that the bases $C=\{(1),(12),(123)\}$ and $D=\{(1),(13),(123)\}$ are both feasible for $v$. So, (12) is a neighbor of $D$. It follows that $(D,(12))$ is a feasible pair for $v$. The induced inequality in $I(v)$ is

$$
v(12) \leq v(1)+v(123)-v(13) \text {. }
$$

Thus, elements of the set $L(v)$ satisfy the equality $v(1)+v(123)=v(12)+v(13) .^{3}$ $\triangleleft$

Derks and Kuipers (2002) show that the core of an $n$-person TU game has at most $n$ ! extreme points. Thus, for any game $v$ for which the core has exactly $n$ ! extreme points the set $L(v)$ is automatically maximal. It might be conjectured that, conversely, a game $v$ whose set $L(v)$ is maximal always has $n$ ! extreme points. The following example from Derks and Kuipers (2002) shows that this is not correct, not even for strict exact games.

Example The symmetric game $w$ on player set $N=\{1,2,3,4,5\}$ is defined by

\begin{tabular}{r|ccccc}
$|S|$ & 1 & 2 & 3 & 4 & 5 \\
\hline$w(S)$ & 0 & 1 & 8 & 11 & 23
\end{tabular}

Derks and Kuipers (2002) show that $w$ is strict exact, and that there are

[1] 20 extreme elements of $C(w)$ with coordinates $0,4,4,4,11$.

[2] 20 extreme elements of $C(w)$ with coordinates $2,3,3,3,12$.

[3] 60 extreme elements of $C(w)$ with coordinates $0,1,7,7,8$.

These are all the extreme core elements, so that $C(w)$ has in total 100 extreme points, strictly less than the maximum of $5 !=120$. We argue that $L(w)$ is maximal. For the allocation $a=(0,4,4,4,11)$,

$$
T(w, a)=\{(1),(123),(124),(134), N\} .
$$

Notice that $T(w, a)$ is a basis. Similarly, for the allocation $b=(2,3,3,3,12)$,

$$
T(w, b)=\{(123),(124),(134),(1234), N\}
$$

is a basis. And, for the allocation $c=(0,1,7,7,8)$,

$$
T(w, c)=\{(1),(12),(123),(124), N\}
$$

is a basis. Then Theorem 5.4 implies that $L(w)$ is maximal.

\footnotetext{
${ }^{3}$ Exact equality of inequalities on sets $L(v)$ that are not maximal linear regions is a general feature that is in fact shown in Lemma 2 of Kuipers et al. (2010).
} 
Open Access This article is distributed under the terms of the Creative Commons Attribution 4.0 International License (http://creativecommons.org/licenses/by/4.0/), which permits unrestricted use, distribution, and reproduction in any medium, provided you give appropriate credit to the original author(s) and the source, provide a link to the Creative Commons license, and indicate if changes were made.

\section{References}

Bondareva ON (1962) Theory of the core in the $n$-person game. Vestnik Leningradskii Universitet 13:141142 (in Russian)

Bloch F, de Clippel G (2010) Cores of combined games. J Econ Theory 145:2424-2434

Derks J, Kuipers J (2002) On the number of extreme points of the core of a transferable utility game. In: Chapters in game theory, in honor of Stef Tijs. Theory and decision library, Series C. Kluwer Academic Publishers, pp 83-97

Kuipers J, Vermeulen D, Voorneveld M (2010) A generalization of the Shapley-Ichiishi result. Int J Game Theory 39:585-602

Shapley LS (1967) On balanced sets and cores. Naval Res Logist Q 14:453-460 